This item was submitted to Loughborough's Research Repository by the author.

Items in Figshare are protected by copyright, with all rights reserved, unless otherwise indicated.

\title{
Development of a high-throughput UHPLC-MS/MS (SRM) method for the quantitation of endogenous glucagon from human plasma
}

\section{PLEASE CITE THE PUBLISHED VERSION}

http://dx.doi.org/10.4155/bio.14.226

\section{PUBLISHER}

(c) Future Science Ltd

\section{VERSION}

AM (Accepted Manuscript)

\section{PUBLISHER STATEMENT}

This work is made available according to the conditions of the Creative Commons Attribution-NonCommercialNoDerivatives 4.0 International (CC BY-NC-ND 4.0) licence. Full details of this licence are available at: https://creativecommons.org/licenses/by-nc-nd/4.0/

\section{LICENCE}

CC BY-NC-ND 4.0

\section{REPOSITORY RECORD}

Howard, James W., Richard G. Kay, Tricia Tan, James Minnion, Mohammad Ghatei, Steve Bloom, and Colin S. Creaser. 2019. "Development of a High-throughput UHPLC-MS/MS (SRM) Method for the Quantitation of Endogenous Glucagon from Human Plasma". figshare. https://hdl.handle.net/2134/26784. 
Development of a high throughput UHPLC-MS/MS (SRM) method for the quantitation of endogenous glucagon from human plasma

James W Howard ${ }^{1,2 \dagger}$, Richard G Kay ${ }^{1}$, Tricia $\operatorname{Tan}^{3}$, James Minnion ${ }^{3}$, Mohammad Ghatei ${ }^{3}$, Steve Bloom ${ }^{3}$ and Colin S Creaser ${ }^{2}$

1 LGC Limited, Newmarket Road, Fordham, Cambridgeshire, CB7 5WW, UK

2 Centre for Analytical Science, Department of Chemistry, Loughborough University, Leicestershire, LE11 3TU, UK

3 Imperial College, Department of Investigative Medicine, Hammersmith Hospital Campus, Du Cane Road, London, W12 0NN, UK † Author for correspondence. Tel: +44 (0) 1638720 500. Fax: +44 (0)1638 724200

Email: james.howard@lgcgroup.com

\section{Abstract}

Background: Published LC-MS/MS methods are not sensitive enough to quantify endogenous levels of glucagon. Results: A UHPLC-MS/MS (SRM) method for the quantitation of endogenous levels glucagon was successfully developed and qualified. A novel 2D extraction procedure was used to reduce matrix suppression, background noise and interferences. Glucagon levels in samples from healthy volunteers were found to agree with RIA derived literature values. Bland-Altman analysis showed a concentration-dependent positive bias of the LC/MS-MS assay versus an RIA. Both assays produced similar pharmacokinetic profiles, both of which were feasible considering the nature of the study.

Conclusions: Our method is the first peer reviewed LC-MS/MS method for the quantitation of endogenous levels of glucagon, and offers a viable alternative to RIA based approaches.

\section{Introduction}

Glucagon is a 29 amino acid peptide which is one of multiple hormones that modulates glucose production or utilisation to regulate blood glucose levels. It is also a biomarker for pathologies such as diabetes, pancreatic cancer or certain neuroendocrine tumours [1]. It is known to be degraded by peptidases such as dipeptidyl peptidase IV [2][3] and consequently blood samples are typically collected in tubes containing protease inhibitors.

Endogenous glucagon levels in healthy patients are reported between $25-80 \mathrm{pg} / \mathrm{mL}$, which may be raised by about $10 \mathrm{pg} / \mathrm{mL}$ in pancreatic cancer patients, and can reach up to 160 
$\mathrm{pg} / \mathrm{mL}$ in diabetic patients [1]. Following treatments using glucagon infusion levels can reach $\sim 906 \mathrm{pg} / \mathrm{mL}$ [4]. Glucagon concentrations are routinely measured using radioimmunoassay (RIA) based approaches, however these assays can be time consuming to perform (up to 3 days) and the kits hasuppleve limited lifetimes (e.g. 2 months). In addition they can suffer from poor precision and accuracy, as there is potential for cross reactivity with similar compounds or inactive degradation fragments leading to inaccurate quantitation [5][6][7]. For example, whilst a comparison between two glucagon immunoassays resulted in a high correlation ( $R=0.97$ ), the concentrations between individual samples differed by 2-4 fold [8]. The radioactive nature of RIAs also necessitates additional health and safety precautions during set-up, and specialised disposal of radioisotopes.

A LC-MS/MS assay would have the potential to circumvent such problems [9], and may offer additional benefits such as a reduced sample volume and a higher throughout. However, published LC-MS/MS methods [10][11] are not sensitive enough to detect endogenous glucagon levels. As described in a recent review paper [12] the lowest reported LLOQ in the peer reviewed literature is $250 \mathrm{pg} / \mathrm{mL}$ [11], although assays of $100 \mathrm{pg} / \mathrm{mL}$ [13] and $10 \mathrm{pg} / \mathrm{mL}$ [14] have been described at recent conferences.

Furthermore, as glucagon is produced endogenously, this presents additional experimental

In the standard addition based approach, analyte is spiked on top of the authentic matrix to challenges as an authentic analyte free matrix cannot be obtained to construct calibration standards. Either a standard addition, surrogate analyte, or a surrogate matrix approach must therefore be used [15][16]. create a calibration line, which is extrapolated to measure concentrations below the matrix's endogenous value. However the USA FDA Guidance for Bioanalytical Method Validation [17] actively discourages the extrapolation of calibration curves beyond their range. The surrogate analyte based approach uses an analogue to the analyte in place of the analyte itself in calibration samples. As this will have a Selected Reaction Monitoring (SRM) transition unique from the authentic analyte these can be prepared in authentic biological matrix [15] . However, this approach requires the relationship between the authentic and surrogate analyte to be thoroughly investigated, the approach is not commonly used, and is not considered in the FDA [17] or EMA guidelines [18]. Alternatively, in the surrogate matrix approach, calibration lines are constructed by spiking analyte into a surrogate matrix. QCs can be prepared in actual sample matrix, and the accuracy calculated to demonstrate the absence of a matrix effect. Surrogate matrices may be the authentic matrix stripped of analyte (e.g. by charcoal [16] or immuno-afffinity methods [19]) or an alternative matrix (e.g. protein buffers, dialysed serum [20]). Although not ideal, the EMA Guideline on bioanalytical 
method validation [18] concedes that such an approach may be necessary for endogenous analyte quantitation, and therefore this is the approach we adopted.

This article outlines the first peer reviewed high throughput UHPLC-MS/MS (SRM) based approach capable of quantifying endogenous levels of glucagon from human plasma. The high throughput nature of the assay is due to its ability to relatively quickly analyse large numbers of samples. This is enabled by an extraction procedure that is relatively quick, simple, and cheap in comparison to many immunochemistry based approaches [21], and which can analyse large number of samples ( 60) within an analytical batch. In addition, UHPLC is used to minimise sample run times [22]. A calibration range of $25-1000 \mathrm{pg} / \mathrm{mL}$ is qualified, making the assay suitable for measuring both endogenous levels of glucagon and elevated levels following treatments. Consequently the assay can be used for both biomarker (PD, Pharacodymaic) and Pharmacokinetic (PK) analysis. However, the calibration range could be easily truncated if only endogenous level analysis (PD) is required. In addition we present the first comparison of glucagon concentrations determined by an LC-MS/MS assay and a traditional RIA method using a large number of clinical samples derived from a physiological study of glucagon's actions in the body $(n=88)$.

The assay's performance has been evaluated using experiments described in the latest EMA [18] and FDA [17] guidance and in accordance to the principles of GCP [23].

\section{Key Terms}

Radioimmunoassay (RIA) - A highly sensitive technique used to measure concentrations of antigens (e.g. peptides) by use of antibodies. Pre-bound radioactively labelled antigens are displaced by non-radioactive antigens from a sample. Monitoring the change in radioactivity allows quantitation.

UHPLC-MS/MS (SRM) - An analytical methodology that combines the use of ultra-high performance liquid chromatographic (UHPLC) separations with sensitive mass spectrometer selected reaction monitoring (SRM). Traditionally used for small molecule quantitation, but increasingly used for the quantitation of biological molecules (e.g. peptides). 


\section{Experimental}

\section{Chemicals and materials}

Certified human glucagon (HSQGTFTSDYSKYLDSRRAQDFVQWLMNT) was obtained from EDQM (Strasbourg, France) and the analog internal standard (IS) (des-thr ${ }^{7}$-glucagon) (HSQGTFSDYS KYLDSRRAQDFVQWLMNT) from Bachem (Bubendorf, Switzerland). This internal standard has given suitable performance in LC-MS/MS glucagon assays [13] [14], and it avoids the expense of synthesising a heavy labelled internal standard. Water was produced by a Triple Red water purifier (Buckinghamshire, U.K.). BD glass collection tubes $(5 \mathrm{~mL})$ containing K3 EDTA anticoagulant and 250 Kallikrein Inhibitor Units (KIU) of Aprotinin were obtained from BD (Oxford, UK). Following collection, tubes were placed on ice, then centrifuged at $2300 \times \mathrm{g}$ for 10 minutes to obtain plasma, which was stored at $-80^{\circ} \mathrm{C}$ when not in use. All chemicals and solvents were HPLC or analytical reagent grade and purchased from commercial vendors.

\section{Instrumentation: LC-MS/MS}

The LC-MS/MS system consisted of a Waters Acquity UPLC system (Waters Corporation, Massachusetts, USA) coupled to an AB SCIEX 5500 QTRAP (Applied Biosystems / MDS SCIEX, Ontario, Canada) with an electrospray ion source. Data acquisition and processing were performed using Analyst 1.5.2 (Applied Biosystems/ MDS SCIEX). The majority of the chromatograms were integrated using fully automated settings. A minority had their integration settings (peak selection, peak splitting factor, noise percentage) altered to ensure appropriate and consistent integration. No samples were integrated using manual integration mode.

Glucagon was separated on a Waters UPLC BEH C18 $1.7 \mu \mathrm{m}(2.1 \times 100 \mathrm{~mm})$ column maintained at $60{ }^{\circ} \mathrm{C}$. The mobile phase consisted of $(A) 0.2 \%$ formic acid (FA) in acetonitrile $(\mathrm{MeCN})$ and $(\mathrm{B})$ 0.2\% FA (aq). The gradient for separation was $22-32 \% \mathrm{~A}$ over 2 minutes. The column was then cleaned with $95 \% \mathrm{~A}$ for approximately 1 minute then $22 \% \mathrm{~A}$ for approximately 4 minutes. The flow rate was $0.8 \mathrm{~mL} / \mathrm{min}$ and the total run time 7.1 minutes.

The mass spectrometer was operated in positive ion mode with an electrospray voltage of $5500 \mathrm{~V}$, an entrance potential of $10 \mathrm{~V}$, and a declustering potential of $70 \mathrm{~V}$. The source temperature was $600^{\circ} \mathrm{C}$, the curtain gas $40 \mathrm{Psi}$, and the desolvation gases, GS1 and GS2, were set at 60 psi and 40 psi respectively. Quantitation was performed using the selected reaction monitoring (SRM) transitions $697.5 \rightarrow 693.8$ and $677.2 \rightarrow 673.8$ for glucagon and the internal standard respectively. The $\mathrm{N}_{2}$ collision gas was set to medium and both transitions 
used collision energies of $15 \mathrm{~V}$ and collision exit cell potentials of $13 \mathrm{~V}$. The Q1 and Q3 quadruples were both operated at unit resolution.

$1311 \mathrm{mg} / \mathrm{mL}$ stock solutions of glucagon and glucagon internal standard were prepared in 132 borosilicate vials using surrogate matrix [Methanol $(\mathrm{MeOH}): \mathrm{H}_{2} \mathrm{O}$ : Formic acid (FA): Bovine serum albumin (BSA), (20:80:0.1:0.1, v/v/v/w)]. Glucagon working solutions were prepared by dilution with this solvent to create nine calibration standard spiking solutions $(125,225$, $375,500,1000,2000,3000,4500,5000 \mathrm{pg} / \mathrm{mL}$ ), and four quality control spiking solutions $(125,250,10000,75000 \mathrm{pg} / \mathrm{mL})$. Additional calibration standard and QC spiking solutions at 75 and $50 \mathrm{pg} / \mathrm{mL}$ were also prepared for the assessment of assay performance at the 10 and $15 \mathrm{pg} / \mathrm{mL}$ levels. Internal standard working solution (ISWS) was similarly prepared at 20 $\mathrm{ng} / \mathrm{mL}$. The stock and working solutions were prepared to a volume of $10 \mathrm{~mL}$ and were stored at $-20^{\circ} \mathrm{C}$ when not in use. QC MED and QC HIGH plasma samples were prepared by diluting the appropriate spiking solution 100 fold with plasma to create samples at 100 and $750 \mathrm{pg} / \mathrm{mL}$ respectively. These were either used immediately, or stored at $-80{ }^{\circ} \mathrm{C}$ prior to use.

\section{Extraction method development \& surrogate matrix quantitation}

Additional details of the extraction method development experiments described are provided in the supplementary information. In summary:

147 Protein precipitation optimisation The following precipitation solvents were investigated; 148 Acetonitrile (MeCN), MeCN: $\mathrm{H}_{2} \mathrm{O}(50: 50, \mathrm{v} / \mathrm{v})$, and $\mathrm{MeCN}: \mathrm{H}_{2} \mathrm{O}(75: 25, \mathrm{v} / \mathrm{v})$. Each solvent was 149 investigated with and without $0.1 \%$ formic acid. In addition $\mathrm{MeCN}: \mathrm{H}_{2} \mathrm{O}: \mathrm{NH}_{3}$ (75:25:0.1, $150 \mathrm{v} / \mathrm{v} / \mathrm{v})$ was investigated.

151 Solid phase extraction optimisation Extraction efficiencies of the MAX, MCX, and WCX 152 phases from a 96 well Oasis sorbent selection plate $(10 \mathrm{mg})$ (Waters Corporation) and from 153 a size exclusion hydrophobic (SEH) Bond Elut Plexa 96 round-well (30 mg) plate (Agilent 154 Technologies, California, USA) were evaluated. The Oasis extraction used generic 155 conditions for peptide analysis based on those provided by the manufacturer, whilst we used our in house generic conditions for the Plexa evaluation.

157 Surrogate matrix quantitation- The calibration standard spiking solutions described above 158 were diluted 5 fold with surrogate matrix. $400 \mu \mathrm{L}$ aliquots were then extracted according to 159 the procedure below. The matrices investigated were $\mathrm{H}_{2} \mathrm{O}, \mathrm{MeOH}: \mathrm{H} O \mathrm{O}: \mathrm{FA}: \mathrm{BSA}$ 160 (20:80:0.1:0.1, v/v/v/w), 6\% BSA (aq) and 6\% rat plasma (aq). 
162 Plasma sample (aprotinin stabilised, K3 EDTA) $(400 \mu \mathrm{L})$ was placed into a $5 \mathrm{~mL}$ polypropylene tube and $20 \mu \mathrm{L}$ of ISWS was added to all non-blank samples. The samples were briefly vortex mixed, precipitated using $3.2 \mathrm{~mL}$ of $\mathrm{MeCN}: \mathrm{H}_{2} \mathrm{O}: \mathrm{NH}_{3}(72: 25: 0.1, \mathrm{v} / \mathrm{v} / \mathrm{v})$, vortex mixed again, and then centrifuged for 10 minutes at $2300 \times \mathrm{g}$. The supernatant was transferred to a new tube and evaporated to dryness overnight under vacuum. Samples were reconstituted in $800 \mu \mathrm{L} 2 \% \mathrm{NH}_{3}$ (aq) and then vortex mixed. A Bond Elut Plexa 96 round-well solid phase extraction (SPE) plate (30 mg) was conditioned using $1 \mathrm{~mL} \mathrm{MeOH}$, then equilibrated with $1 \mathrm{~mL} \mathrm{H}_{2} 0$. The samples were loaded, washed with $1 \mathrm{~mL} 5 \% \mathrm{MeOH}$ (aq), eluted with $2 \times 225 \mu \mathrm{L}$ MeCN: $\mathrm{H}_{2} \mathrm{O}: \mathrm{FA}(75: 25: 0.1, \mathrm{v} / \mathrm{v} / \mathrm{v})$, and then evaporated under nitrogen at $40^{\circ} \mathrm{C}$, before being reconstituted in $200 \mu \mathrm{L} 0.2 \% \mathrm{FA}(\mathrm{aq})$.

Calibration standards, QC LLOQs and QC LOWs were then prepared freshly for each batch by spiking $80 \mu \mathrm{L}$ of the appropriate spiking solution into the plate, along with $20 \mu \mathrm{L}$ of ISWS and $100 \mu \mathrm{L}$ surrogate matrix. Taking into account the 2 -fold concentration experienced by plasma samples ( $400 \mu \mathrm{L}$ of plasma sample is reconstituted into $200 \mu \mathrm{L}$ of solvent) this gives final calibration levels of $25,45,75,100,200,400,600,900$, and $1000 \mathrm{pg} / \mathrm{mL}$, and final QC levels of 25 and $50 \mathrm{pg} / \mathrm{mL}$. The plate was centrifuged for 10 minutes at $2300 \times \mathrm{g}$, and $50 \mu \mathrm{L}$ of sample injected on to the LC-MS/MS system for analysis.

\section{Validation Experiments}

The validation experiments chosen were based on those described in the latest EMA guidance [18]. Calibration standards were analysed in duplicate with each batch. Data was imported into Watson LIMS 7.2 (Thermo Fisher Scientific Inc, Massachusetts, USA) and linear regression with $1 / x^{2}$ weighting was applied to the peak area ratios-concentration plot for the construction of calibration lines. The precision and accuracy of the method was determined by analysis of replicate $(n=6)$ QC samples at four different concentrations (25, 50,100 , and $750 \mathrm{pg} / \mathrm{mL}$ ), and was assessed within a batch (intra-batch, $\mathrm{n}=6$ replicates) and between batches (inter-batch, 3 batches). The ability to dilute was assessed by diluting an over range dilution sample $(7500 \mathrm{pg} / \mathrm{mL}) 10$-fold with blank plasma. Carryover effects were evaluated by injection of blank samples immediately after injection of the highest point in the calibration range.

Selectivity was assessed by qualitatively examining chromatograms from six independent control matrix samples for the presence of potentially interfering peaks. It was not feasible to monitor multiple charge states or SRM transitions to further ensure selectivity as only the selected transition demonstrated sufficient sensitivity at the endogenous concentration. The 
modification of analyte and internal standard responses to the presence of matrix was also determined in such samples. These were extracted and post spiked at either the medium or high level, and compared to the mean response from samples in surrogate matrix (minimum $\mathrm{n}=6)$. The effect of haemolysed (3\%) plasma and hyperlipidaemic plasma $(\sim 4 \mathrm{mmol} / \mathrm{L}$ of triglycerides) upon on quantitation was investigated by preparing QCs in these matrices at the medium and high level ( $n=6$ replicates). Recovery of the analyte was evaluated by comparing the analytical results for extracted analyte samples at the medium and high level with unextracted analyte samples that represent $100 \%$ recovery.

The stability of the glucagon in aprotinin stabilised human plasma was evaluated at the medium and high concentrations in replicate $(n=6)$. Stability was assessed after $6 \mathrm{hr} 20 \mathrm{~min}$ on ice $\left(4^{\circ} \mathrm{C}\right)$, after storage for 11 and 75 days at $-20^{\circ} \mathrm{C}$, and for $7,11,51$, and 64 days at $-80^{\circ} \mathrm{C}$. Similarly stability was assessed after 4 freeze-thaw cycles from $-20^{\circ} \mathrm{C}$ to $4{ }^{\circ} \mathrm{C}$ and also 4 freeze cycles from $-80^{\circ} \mathrm{C}$ to $4^{\circ} \mathrm{C}$. Stability was similarly assessed in whole blood following storage on ice for 1 hour. The ability to re-inject sample extracts at medium and high concentrations was assessed after storage at $+4^{\circ} \mathrm{C}$ for 6 days. The stability of the stock solution was assessed after storage at $-20^{\circ} \mathrm{C}$ for 66 days and that of LLOQ and ULOQ working solutions after 163 days at $-20^{\circ} \mathrm{C}$.

All results are quoted from batches where the standards and QCs passed our prospectively defined acceptance criteria, which were based on the EMA and FDA guidelines. These required that at least $75 \%$ of standards in each batch had back calculated accuracy within $15 \%$ (20\% at the LLOQ) of the nominal concentration, with standards outside these criteria excluded from the regression. QCs in precision and accuracy batches needed to have mean intra-batch accuracy within $20 \%$ of the nominal concentration, and intra-batch precision that did not exceed $20 \%$. In other batches at least $2 / 3$ of the individual QCs had accuracy within $20 \%$ of the nominal concentration, with at least one QC passing criteria at each level. Although the guidelines suggest a $15 \%$ criteria (20\% at the LLOQ) should be applied to QC performance, they state it can be widened prospectively in special cases. We felt it was justified to raise the QC acceptance criteria to $20 \%$ (CV and RE) due to the surrogate matrix nature of the assay. The $20 \%$ (RE) acceptance criteria was also applied to plasma, blood and extract stability experiments, as well as to the assessment of the matrix effect in different individuals (matrix factor ratio) and of the effect of haemolysed or hyperlipidaemic plasma. 
231 Plasma was collected from 12 healthy males and 12 healthy females using glass collection

232 tubes containing K3 EDTA and aprotinin, as described above. Glucagon levels were 233 determined using the qualified LC-MS/MS method. Plasma was collected at the start of the 234 working day and volunteers were not asked to change their usual eating regime.

\section{Collection of physiological study samples}

237 Physiological study samples $(n=117)$ were collected by Imperial College London. The 238 samples originated from 7 different individuals who were each infused with a glucagon 239 solution at either 16 or $20 \mathrm{pmol} / \mathrm{kg} / \mathrm{min}$ for 12 hours subcutaneously. Blood samples at 240 various time points were collected in $5 \mathrm{~mL}$ lithium heparin collection tubes containing 1000 $241 \mathrm{KIU}$ of Aprotinin, spun down in a cold centrifuge within 5 to 10 mins of collection, and then 242 stored at $-20^{\circ} \mathrm{C}$.

\section{Analysis of physiological study samples}

244 A selection of the physiological study samples $(n=100)$ were analysed by LGC using the LC245 MS/MS method described above. Additional QCs prepared in aprotinin stabilised plasma 246 with lithium heparin anticoagulant were analysed to ensure assay performance in the sample 247 matrix. 38 of the study samples were analysed over the calibration range $25-1000 \mathrm{pg} / \mathrm{mL}$, 248 whilst the remainder were analysed over the calibration range $10-1000 \mathrm{pg} / \mathrm{mL}$. For these 249 samples additional calibration points and QCs were included at the 10 and $15 \mathrm{pg} / \mathrm{mL}$ levels 250 to evaluate assay performance. Samples $(n=105)$ were also analysed by Imperial College 251 using their established radioimmunoassay method over the calibration range $5-1000 \mathrm{pg} / \mathrm{mL}$, 252 which is directed against the C-terminal region of glucagon [24][25]. Samples were analysed upon their first freeze-thaw.

\section{Results and discussion}

\section{Method development}

257 Analysis of endogenous levels of glucagon by LC-MS/MS poses a significant technical 258 challenge. Not only are the low endogenous concentrations difficult to measure, an 259 endogenous analyte quantitation strategy must be used, and stability issues must be 260 addressed. 
261 Extensive assay optimisation was therefore performed to obtain the low $25 \mathrm{pg} / \mathrm{mL}$ LLOQ. A

262 QTRAP mass spectrometer was used in SRM mode, and parameters were optimised.

263 UHPLC was chosen for chromatographic separation because it results in greater efficiencies

264 [26] and/or shorter runtimes [27] than the HPLC commonly used for such separations. The 265 greater efficiency can lead to lower matrix effects due to improved separation from matrix 266 suppressants [28] and to higher sensitivities due to sharper peak shapes [22].

267 The $\left[\mathrm{M}+5 \mathrm{H}^{+}\right]^{5+}$ ion was found to give the highest intensity during MS method development 268 (Figure 1a), although other studies have found the $\left[\mathrm{M}+4 \mathrm{H}^{+}\right]^{+4}$ to be optimal [11][10]. MS2 269 experiments showed that showed that the ionic species generated by ESI of glucagon were 270 able to absorb substantial collision energy without undergoing major fragmentations, as 271 demonstrated previously [10] (Figure 1b). As also reported [13][12] an SRM transition 272 corresponding to the loss of ammonia $\left(\left[\mathrm{M}+5 \mathrm{H}^{+}\right]^{+5} /\left[\mathrm{M}+5 \mathrm{H}^{+}-\mathrm{NH}_{3}\right]^{+5}\right.$ was found to be optimal. 273 Although this is not a particularly specific transition, the intensity was significantly greater 274 than other transitions and was therefore chosen; selectivity was fully investigated during the 275 validation. Resolution settings for Q1 and Q3 were optimal at unit-unit, rather than high-high 276 as reported by others [11]. The optimal ion pairs of the transitions were 697.5/693.8, which 277 corresponds to a $18.5 \mathrm{Da}$ loss. The small difference between our optimal pair, and that 278 previously reported (697.6/694.2) [13][12] is attributed to the resolution limitations of the 279 mass spectrometer used [29], as is the difference between the theoretical mass loss of 280 ammonia (17 Da) and that observed (18.5 Da).

281
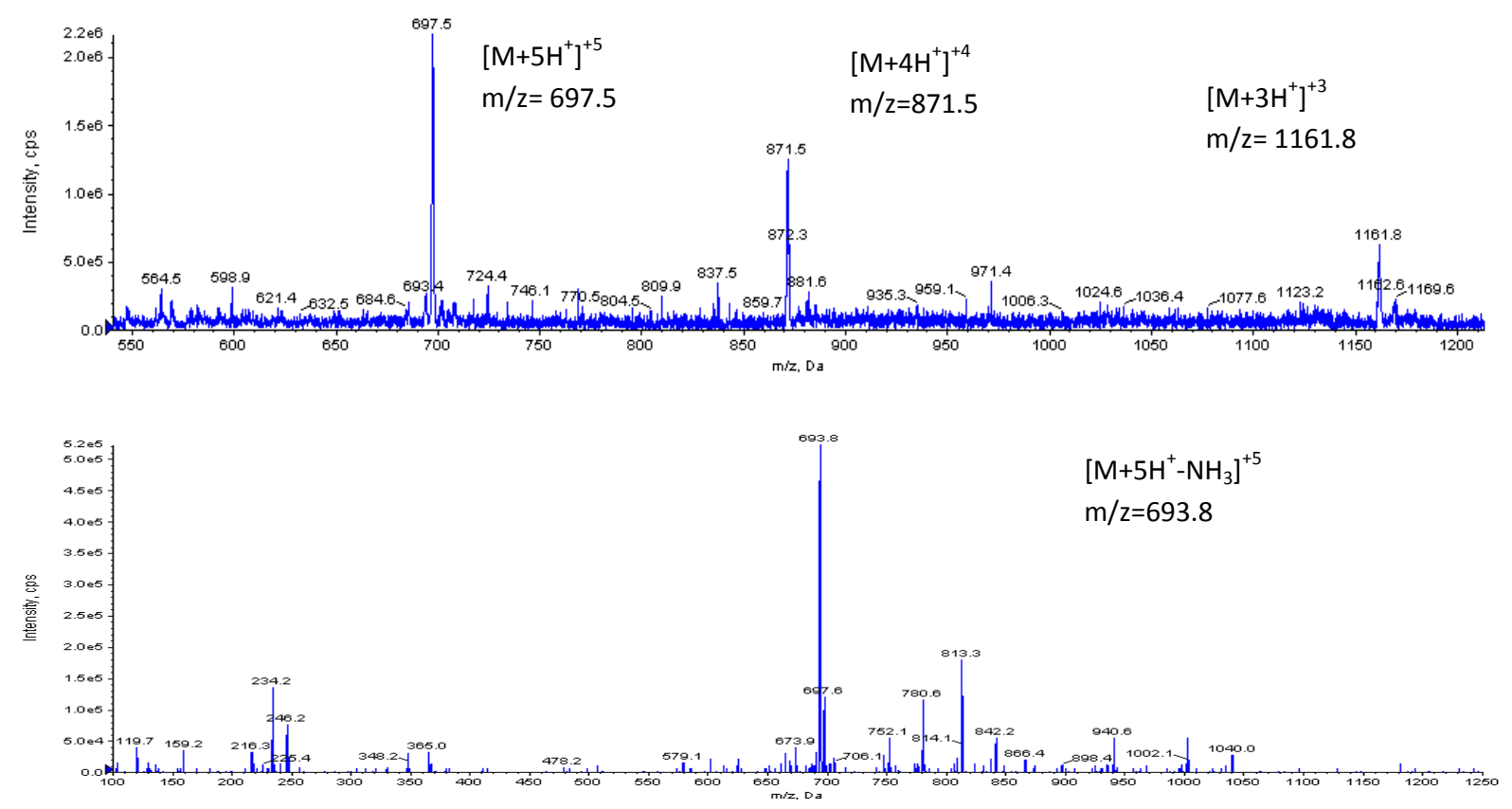
b)(bottom) MS spectrum of production ion scans (Parent $=697.5, \mathrm{CE}=25 \mathrm{~V})$ 
A relatively large $400 \mu \mathrm{L}$ plasma volume was chosen for extraction, to enable concentration of extracts to achieve higher sensitivities. The volume does, however, compare well to the $2 \times 200 \mu \mathrm{L}$ typically required for RIA methods. Initially, protein precipitation based extraction techniques were investigated, as they are quick and cheap, and are amenable to automation and high throughput analysis. Additionally, pure acetonitrile precipitation has been previously selected for glucagon extractions [10] [11]. We have previously demonstrated that diluting acetonitrile with various proportions of water can lead to more specific extractions [30], as can the addition of acids or bases to due to the differences between the isoelectric points $(\mathrm{pl})$ of the proteins or peptides of interest and the background proteins [31]. Precipitation solvents containing various proportions of acetonitrile, water, acid and base were investigated, with $\mathrm{MeCN}: \mathrm{H}_{2} \mathrm{O}: \mathrm{NH}_{3}(75: 25: 0.1, \mathrm{v} / \mathrm{v} / \mathrm{v})$ giving the best response. However, in all cases background noise and interferences were relatively high, as was matrix suppression.

It was therefore decided to investigate solid phase extraction (SPE) based approaches, as these should lead to cleaner samples with reduced background noise and interferences. These studies are described in the supplementary information.

Combining protein precipitation with size exclusion hydrophobic (SEH) SPE was found to reduce the on column matrix effects, whilst providing adequate recovery. To our knowledge this is the first time protein precipitation has been combined with SEH SPE for quantitative peptide analysis, although protein precipitation has been combined with other SPE phases for this purpose[32]. Due to the satisfactory performance of this extraction methodology, alternatives such as immunoaffinity enrichment were not investigated [33].

Various UHPLC gradients were investigated to further reduce matrix build-up on the column and it was found that a 4 minute flush at the starting conditions gave the best performance. This gradient combined with the 2D extraction methodology significantly increased the robustness of the assay.

312 Glucagon is known to be degraded by the blood enzymes and consequently sample stabilisation is required [2] . The enzyme inhibitor aprotinin was used to reduce degradation and samples were extracted on ice. As there have been reports of enzyme inhibitors interfering with peptide quantitation [34] assay performance was closely monitored during the validation for any such issues. 
319 Several mixtures were screened for their suitability as surrogate matrices. A dilute buffer 320 matrix was evaluated, as such matrices have been shown to be suitable for some assays. 321 [35] [19]. A buffer solution containing a relatively high percentage of BSA was also evaluated 322 to minimise any non-specific analyte binding that may occur. In addition a diluted rat plasma 323 matrix was chosen to investigate whether biological matricies improved assay performance.

324 The dilute buffer matrix, Water and $\mathrm{MeOH}: \mathrm{H}_{2} \mathrm{O}: \mathrm{FA}$ : BSA (20:80:0.1:0.1, v/v/v/w), resulted in low signals following extraction, which is attributed to non-specific binding of glucagon to plastic consumables used during the extraction procedure, as has been described previously [10]. The $6 \%$ BSA (aq) matrix, selected to minimise non-specific binding in solvent led to a very high background noise, whilst the $6 \%$ rat plasma (aq) led to poor calibration line accuracy against prepared concentrations. It was therefore decided to use $\mathrm{MeOH}: \mathrm{H}_{2} \mathrm{O}$ : FA: BSA (20:80:0.1:0.1, v/v/v/w) as the surrogate matrix, but not to extract samples prepared in this, in order to prevent large losses by nonspecific binding. Whilst plasma samples require extraction, their high protein content prevents binding and the use of an internal standard was expected to take into account recovery differences between the surrogate matrix calibrants (which will necessarily have recovery of $100 \%$ for the analyte and IS) and the extracted plasma samples. The internal standard was also expected to take in to account the differences in matrix effect between the two matrices, as well as any small losses that occurred due to non-specific binding that occurred in the injection plate. Whilst the buffer solution selected as the surrogate matrix is of quite a different nature to the plasma samples, assays for small [35] and large molecules [19] have been successfully validated using such an approach, and the validation experiments described later in this manuscript fully assess the assay's performance. It was decided to proceed with this approach rather than investigate alternative matrices such as charcoal stripped plasma. It has been suggested that when a surrogate matrix approach is used that aliquots of the authentic matrix containing the endogenous analyte should be used as QC MED samples and QC HIGH samples should be prepared by spiking analyte in addition to this endogenous level [35].QC LOW samples are then made by diluting authentic matrix with surrogate matrix, and QC LLOQ samples prepared in pure surrogate matrix. Unfortunately this strategy cannot be used for glucagon quantitation due to its relatively low endogenous levels ( LLOQ to $\sim 3 x$ 349 LLOQ). It was therefore decided to construct QC LOW using surrogate matrix, and QC MED 350 and QC HIGH samples were prepared by spiking analyte on top of the endogenous level in authentic matrix. Due to the low endogenous levels it was decided to limit the LOW level to 2 $x$ LLOQ (rather than the 3x LLOQ typically used [18]. 
353 Human plasma (K3 EDTA) from a commercial supplier was analysed using the assay to 354 determine its suitability as an authentic matrix. As shown in Supplemental Figure 4 such 355 plasma has a significantly raised background compared to plasma collected from volunteers 356 in house. This may be a result of the lack of stabiliser upon collection, the age of the plasma 357 and/or storage conditions. The raised background makes it unsuitable for the construction of 358 QC samples, and therefore it was decided to use plasma collected in house as the integrity 359 of these samples could be ensured. Similarly, sample collection and storage regimes for any clinical samples should be carefully controlled to ensure their integrity.

\section{$361 \quad$ Validation}

362 The precision and accuracy of the method was determined by analysis of replicate $(n=6)$ QC 363 samples at four different concentrations (25, 50, 100 and $750 \mathrm{pg} / \mathrm{mL}$ ). Precision and 364 accuracy was assessed within a batch (intra-batch, $n=6$ replicates) and between batches 365 (inter-batch, 3 batches). The intra- and inter-assay precision did not exceed $20 \%$, nor did the 366 intra- and inter-assay accuracy demonstrating the method was performing robustly 367 (Supplemental Table 1). No carryover after high calibration standards was observed and no 368 potentially interfering peaks were observed during the selectivity assessment. The 10-fold 369 dilution of an over range QC sample $(7500 \mathrm{pg} / \mathrm{mL})$ with control plasma was used to demonstrate the absence of dilution effects (Supplemental Table 2). 
373 The analogue Internal standard (IS) compensated for differences in suppression observed

374 by the analyte in different matrices, with mean matrix factor (MF) ratios being 1.08 and 1.05

375 at the medium and high level; a perfect correction would have a ratio of 1 (Supplemental

376 Table 3).

377 Recovery was assessed across three different batches with a minimum of 3 replicates at 378 each level. In order to investigate whether the nature of the matrix affected recovery it was 379 assessed from; samples where the analyte was spiked into control matrix then immediately 380 extracted, samples where the analyte was spiked into 3 freshly acquired matrix pools then 381 immediately extracted, and finally from samples where the analyte was spiked into matrix 382 then stored for a week at $-80^{\circ} \mathrm{C}$ before extraction (Supplemental Table 4). No significant 383 difference between these experiments was observed, which gave an average analyte 384 recovery of $51.2 \%$ 
Acceptable sensitivity is usually demonstrated by assessing whether the analyte response at the LLOQ level is at least 5 times [18] the average response due to background noise (Figure 2), which was the case for all accepted batches. It is then assumed that an unknown sample at the LLOQ concentration would also have a similarly acceptable response. However, this will not necessarily be the case for surrogate matrix assays, due to differences in the recovery and matrix factor between the surrogate and authentic matrices. By taking into account the mean analyte recovery (51.2\%) and mean matrix factor (0.746) for our assay, it was calculated that signal-to-noise $(\mathrm{S} / \mathrm{N})$ at the LLOQ should be at least 13.1 to ensure that $\mathrm{S} / \mathrm{N}$ for an authentic sample at the LLOQ level $\geq 5$ (assuming an unchanged background level). This criterion was not formally part of our validation, but it was met by all accepted batches.
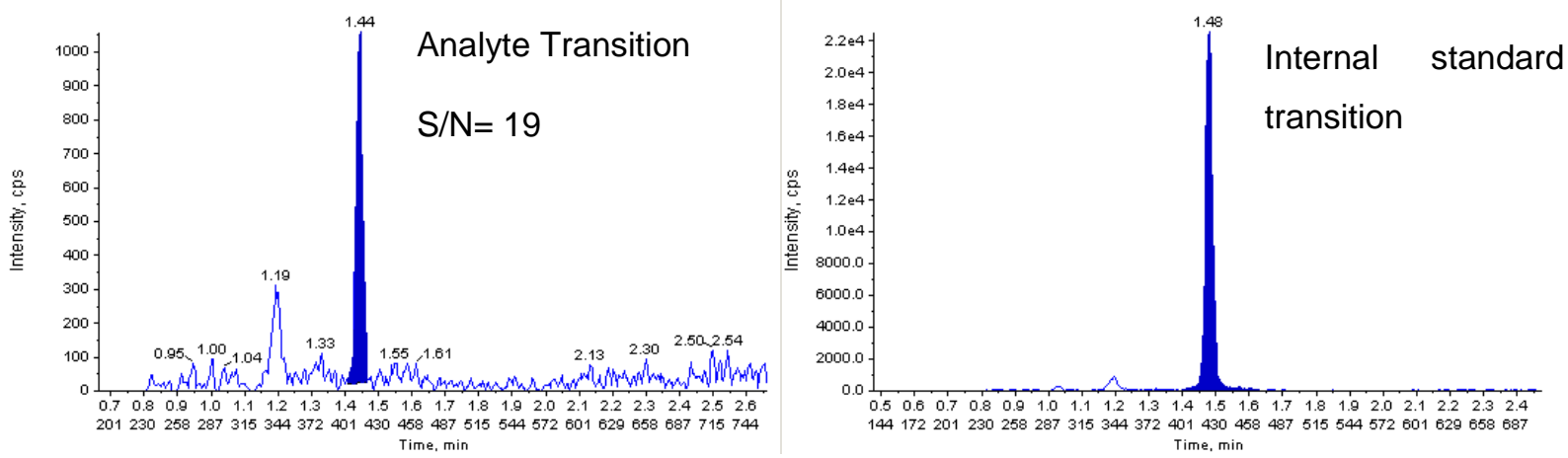

Figure 2- Representative LLOQ for glucagon in plasma $(25 \mathrm{pg} / \mathrm{mL})$ surrogate matrix chromatogram demonstrating a signal-to-noise of $\geq 13.1$

Although we used Aprotinin, a degree of glucagon instability within human plasma was apparent and most experiments gave results outside the acceptance criteria of $\pm 20 \%$ of the nominal concentration (Table 1). Even if $0 \mathrm{hr}$ concentrations were used, to take into account any assay bias or preparation differences, many results remain outside $\pm 20 \%$ of this concentration. Glucagon plasma samples were found to be within $23.7 \%$ of their nominal concentrations following storage at the extraction temperature $\left(+4^{\circ} \mathrm{C}\right)$ for 6 hours 20 minutes, and within $21.4 \%$ of their $0 \mathrm{hr}$ concentration following storage for 75 days at $-20^{\circ} \mathrm{C}$, or within $20.2 \%$ following storage for 51 days at $-80^{\circ} \mathrm{C}$. Greater instability was observed following multiple freeze-thaw cycles, and these should therefore be minimised during analysis. The accuracy of the method is therefore limited by the sub-optimal sample stabilisation procedure. The effect of such pre-analytical parameters has been described by others [36] , and future assay development should include an evaluation of these. For example, stability would likely be improved if specific DPP-IV inhibitors were used [37], rather than the broad serine protease inhibitor Aprotinin. 
416 As stability in Human K3 EDTA plasma with Aprotinin stabilisation did not pass our

417 acceptance criteria, the method is described as qualified, rather than validated. However, the 418 instability was moderate, and the data generated is likely to "fit for purpose" for many 419 applications.

420

\section{Key Terms}

422 Validated assay -An assay where experiments based on those described in the USA FDA 423 Guidance for Industry: Bioanalytical Method Validation (2001) and those described in the 424 EMA Guideline on Bioanalytical Method Validation (2012) meet their prospectively defined 425 acceptance criteria.

426 Qualified assay - An assay where not all of the validation experiments described in the 427 guidance have been assessed or have passed their prospectively defined acceptance 428 criteria. However the assay may still be considered "fit-for-purpose".

429 Fit-for-purpose assay- An assay where its performance characteristics have been assessed 430 and are reliable for the intended application. For example, a biomarker assay which is used 431 to assess a sole pharmacodynamic end point requires better performance characteristics 432 than an assay used as part of a panel of measurements. 
Table 1- Glucagon stability data; Freezer and, extraction temperature stability of glucagon in plasma

\begin{tabular}{|c|c|c|c|c|c|c|c|c|c|c|}
\hline \multirow{3}{*}{$\begin{array}{c}\text { Nominal } \\
\text { Concentration }\end{array}$} & & \multicolumn{9}{|c|}{ Stability of Glucagon in Aprotinin stabilised human plasma (K3 EDTA) } \\
\hline & & \multirow{2}{*}{$\begin{array}{c}+4^{\circ} \mathrm{C} \\
6 \mathrm{hr} 20 \mathrm{~min}\end{array}$} & \multicolumn{3}{|c|}{$-20^{\circ} \mathrm{C}$} & \multicolumn{5}{|c|}{$-80^{\circ} \mathrm{C}$} \\
\hline & & & $4 \mathrm{~F} / \mathrm{T}$ & 11days & 75days & $4 \mathrm{~F} / \mathrm{T}$ & 7days & 11days & 51Days & 64days \\
\hline \multirow{5}{*}{$\begin{array}{c}\text { MED } \\
(100 \mathrm{pg} / \mathrm{mL})\end{array}$} & Mean Measured Conc. (pg/mL) & 76.9 & 54.8 & 83.6 & 81.8 & 75.0 & 89 & - & 81.4 & 71.4 \\
\hline & SD & 4.23 & 6.48 & 6.75 & 5.35 & 5.23 & 5.16 & - & 8.97 & 4.16 \\
\hline & $\% \mathbf{C V}$ & 5.5 & 11.8 & 8.1 & 6.5 & 7.0 & 5.8 & - & 11 & 5.8 \\
\hline & \% Stability (c.f. nominal) & 76.9 & 54.8 & 83.6 & 81.8 & 75.0 & 89.0 & - & 81.4 & 71.4 \\
\hline & \% Stability (c.f. Ohr) & - & 51.6 & 85.5 & 83.7 & 70.6 & 91.0 & - & 81.7 & 71.7 \\
\hline \multirow{5}{*}{$\begin{array}{c}\text { HIGH } \\
\text { (750 pg/mL) }\end{array}$} & Mean Measured Conc. (pg/mL) & 572 & 332 & 581 & 526 & 464 & 530 & 615 & 533 & 445 \\
\hline & SD & 9.50 & 25.3 & 21.9 & 52.8 & 57.7 & 11.9 & 32.7 & 46 & 30.6 \\
\hline & $\% \mathrm{CV}$ & 1.7 & 7.6 & 3.8 & 10 & 12.4 & 2.2 & 5.3 & 8.6 & 6.9 \\
\hline & \% Stability (c.f. nominal) & 76.3 & 44.3 & 77.5 & 70.1 & 61.9 & 70.7 & 82.0 & 71.1 & 59.3 \\
\hline & \% Stability (c.f. Ohr) & 85.6 & 41.5 & 86.8 & 78.6 & 58.0 & 79.2 & 91.9 & 79.8 & 66.7 \\
\hline
\end{tabular}

435

436 SD Standard deviation

CV Coefficient of variation

No data available

$\%$ Stability $($ c.f. nominal) $=100 *$ mean measured concentration $/$ nominal concentration

$438 \%$ Stability (c.f. $0 \mathrm{hr}$ ) $=100 *$ mean measured concentration / mean measured $0 \mathrm{hr}$ concentration

439 Statistics are of $n=6$ replicates, expect for 64 days $\left(-80^{\circ} \mathrm{C}\right)$, which have $n=4$ and $n=5$ replicates at the MED and HIGH level respectively. 
440 The ability to re-inject extracts was demonstrated after storage at $+4^{\circ} \mathrm{C}$ for 6 days

441 (Supplemental Table 5). The stability of stock and working solutions of glucagon, which were

442 stored at $-20^{\circ} \mathrm{C}$ when not in use, was demonstrated for 67 and 163 days respectively

443 (Supplemental Table 6).

444 The stability of glucagon in Aprotinin stabilised whole blood following storage on ice for 1 hour was found to be within acceptance criteria (Supplemental Table 7).

Haemolysed samples (plasma spiked with $3 \%$ whole blood) contained a large neighbouring peak, and did not pass acceptance criteria, demonstrating haemolysed samples cannot be accurately quantified using this method (Supplemental Figure 5). The presence of hyperlipidaemic plasma did not significantly affect the quantitation of glucagon (Supplemental Table 8).

Using the qualified LC-MS/MS method to assess endogenous glucagon concentrations from volunteers

Plasma was collected from 12 healthy males and 12 healthy females and glucagon levels determined using the qualified LC-MS/MS method. As shown in Table 2 levels agreed well with the $25-80 \mathrm{pg} / \mathrm{mL}$ range determined by RIA [1]. Chromatograms from samples which gave glucagon concentrations above the LLOQ showed good signal to noise ratios (Figure 3). Some samples which gave glucagon concentrations below the LLOQ showed integratable peaks (Figure 3) and their approximate concentrations were determined by extrapolation (Table 2)

Table 2- Glucagon concentrations from healthy volunteers.

\begin{tabular}{|c|c|c|c|}
\hline $\begin{array}{c}\text { Male } \\
\text { Volunteer } \\
\text { ID }\end{array}$ & $\begin{array}{c}\text { Measured } \\
\text { glucagon } \\
\text { concentration } \\
\text { (pg/mL) }\end{array}$ & $\begin{array}{c}\text { Female } \\
\text { Volunteer } \\
\text { ID }\end{array}$ & $\begin{array}{c}\text { Measured } \\
\text { glucagon } \\
\text { concentration } \\
\text { (pg/mL) }\end{array}$ \\
\hline M1 & 34.2 & F1 & BLQ (10.4) \\
\hline M2 & 27.4 & F2 & BLQ (16.5) \\
\hline M3 & BLQ (16.0) & F3 & BLQ (12.1) \\
\hline M4 & 31.2 & F4 & 41.6 \\
\hline M5 & 50.2 & F5 & BLQ $(17.7)$ \\
\hline M6 & 63.0 & F6 & 44.4 \\
\hline M7 & BLQ $(21.3)$ & F7 & 29.6 \\
\hline M8 & 53.7 & F8 & 59.5 \\
\hline M9 & 40.4 & F9 & 31.7 \\
\hline M10 & 39.4 & F10 & BLQ \\
\hline M11 & BLQ $(20.0)$ & F11 & BLQ \\
\hline M12 & 153 & F12 & BLQ \\
\hline
\end{tabular}



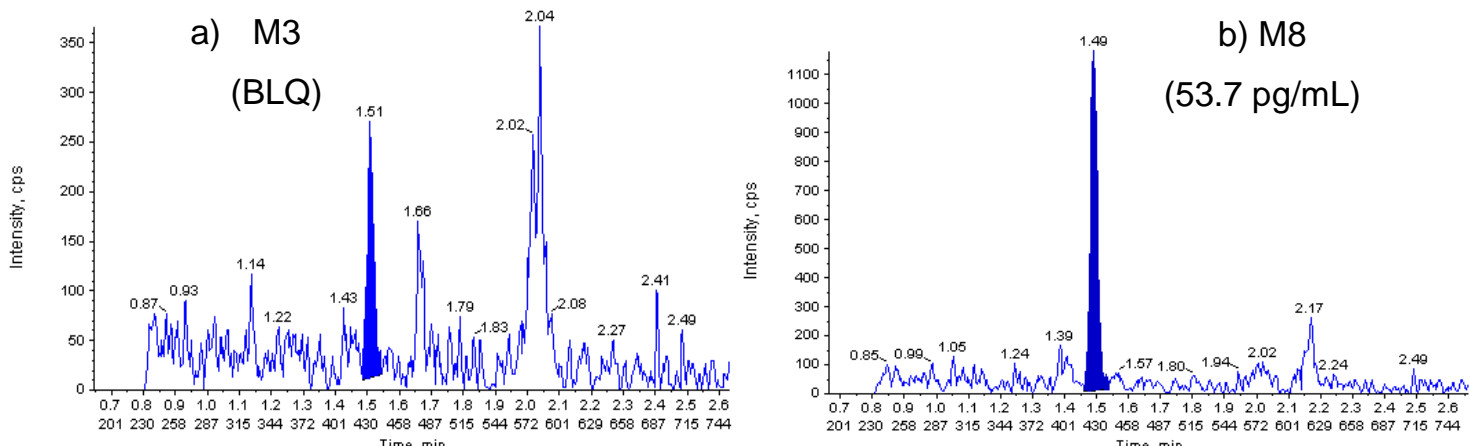
b) $\mathrm{M} 8$ Time, min Time, min

467

468

469

470

471

472

473

474

475

476

477

478

479

480

481

482

483

484

485

486

487
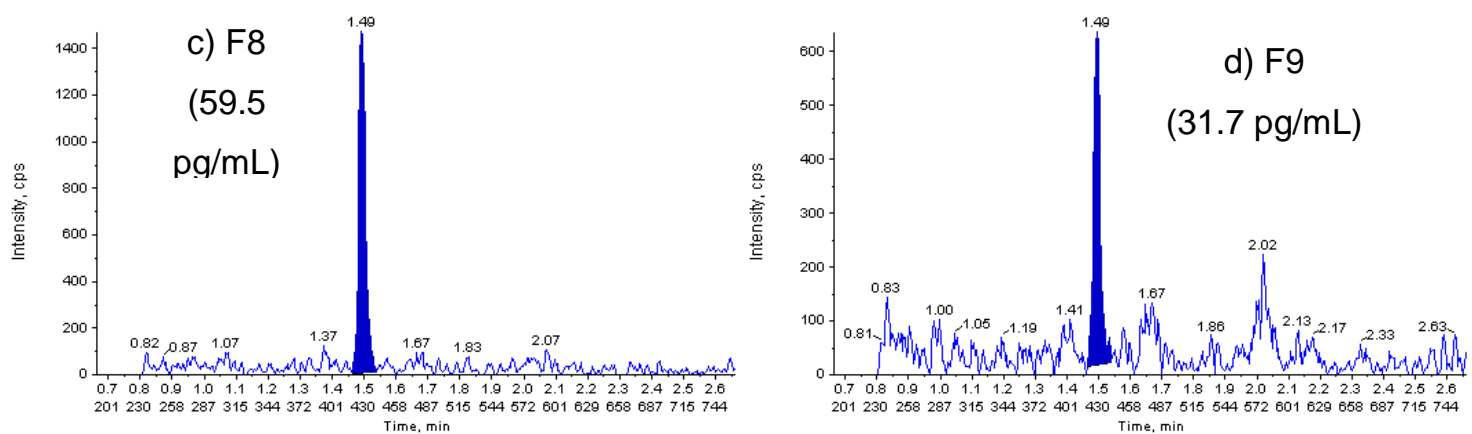

Figure 3 Chromatograms showing endogenous levels of glucagon in plasma samples from healthy volunteers.M3 (a),M8 (b), F8 (c), and F9 (d)

The majority of samples (58\%) gave glucagon concentrations above the $25 \mathrm{pg} / \mathrm{mL}$ qualified LLOQ, demonstrating the assay's utility for endogenous level analysis. However as glucagon concentrations in some individual plasmas were very close to, or below, this level, for subsequent analysis we decided to include additional standards and QCs at the 10 and 15 $\mathrm{pg} / \mathrm{mL}$ concentrations. These allowed assessment of whether a lower LLOQ could be achieved on a batch to batch basis. The acceptable LLOQ was experimentally determined by ensuring that its performance was within acceptance criteria (signal to noise $>5$, and CV and $\operatorname{RE}(<20 \%)$.

To assess whether quantitation was reproducible at the endogenous level, samples containing endogenous glucagon were pooled together, and analysed multiple times in 3 different batches ( $n=6$ replicates in each batch) using the approach above. An overall mean of $26.5 \mathrm{pg} / \mathrm{mL}$ was observed with an overall CV of $19.8 \%$, demonstrating reproducible quantification at the endogenous level (Supplemental Table 9). QCs ( $n=6$ replicates) consistently performed within $20 \%$ (RE and CV) at the $15 \mathrm{pg} / \mathrm{mL}$ level in each of the 3 batches, and were within 20\% (RE and CV) at the $10 \mathrm{pg} / \mathrm{mL}$ level in 2 out of the 3 batches (Supplemental Table 10). This allowed the LLOQ to be reduced from the $25 \mathrm{pg} / \mathrm{mL}$ level in the qualified assay, to increase the proportional of quantifiable concentrations. 
489 Plasma samples $(n=117)$ were collected from a physiological study involving the infusion of 490 glucagon. 100 of these samples were analysed using our LC-MS/MS assay and 105 491 samples using the established RIA assay. Both assays contained QC samples, which 492 performed within their established acceptance criteria.

493 Bland-Altman analysis of the 88 common samples shows that the mean bias of the LC/MS494 MS assay versus the RIA is $+45.06 \mathrm{pg} / \mathrm{ml}$ with $95 \%$ bias confidence intervals of -358.5 to $495448.6 \mathrm{pg} / \mathrm{ml}$. Inspection of the plot (Figure 4 a) shows that there is a concentration496 dependent positive bias, particularly at values above $600 \mathrm{pg} / \mathrm{ml}$, which is also evident in the 497 scatter plot (Figure 4 b) This would be expected if the RIA assay was suffering from the 498 hook effect at higher concentrations, which has been reported for other biomarkers such as 499 calcitonin [38].

500

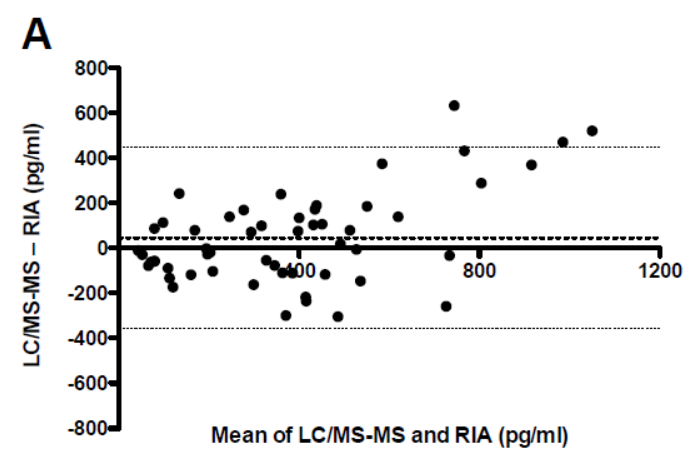

B

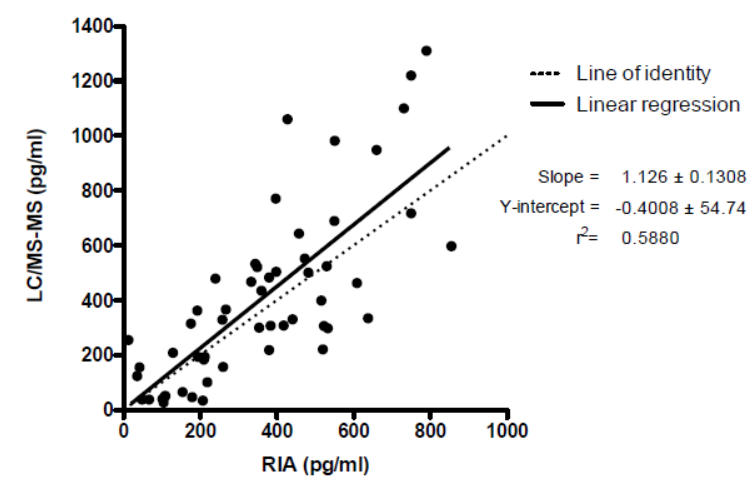

501

502

Figure $4 a-$ Bland-Altman plot and b) scatter plot comparing performance of LC-MS/MS and RIA

505 methods for glucagon.

RIA and LC-MS/MS assays produced pharmacokinetic (PK) profiles of similar shapes, which fitted with expectations from the nature of the study (Figure 5). It is therefore not possible to determine which assay gives the "right" answer, and the approaches should be regarded as complementary. 
Volunteer 1

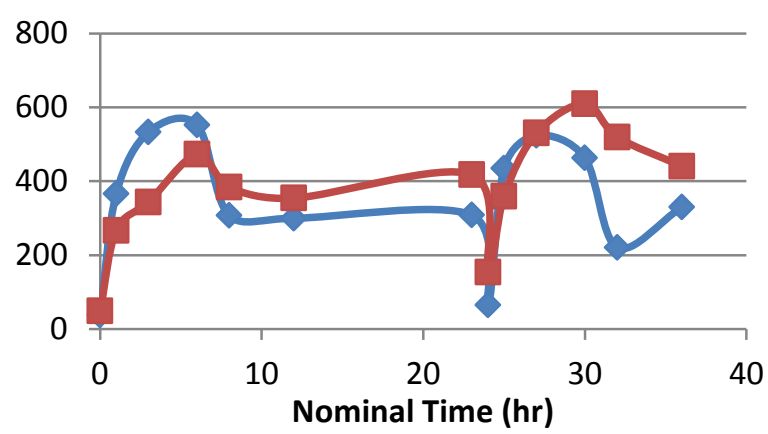

Volunteer 3

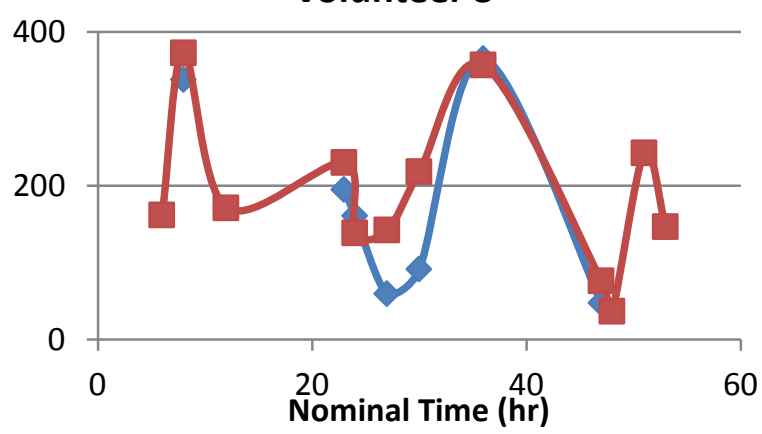

Volunteer 2

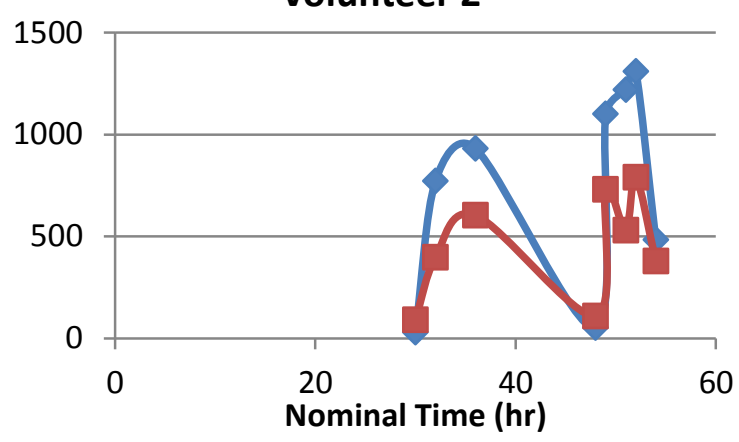

Volunteer 4

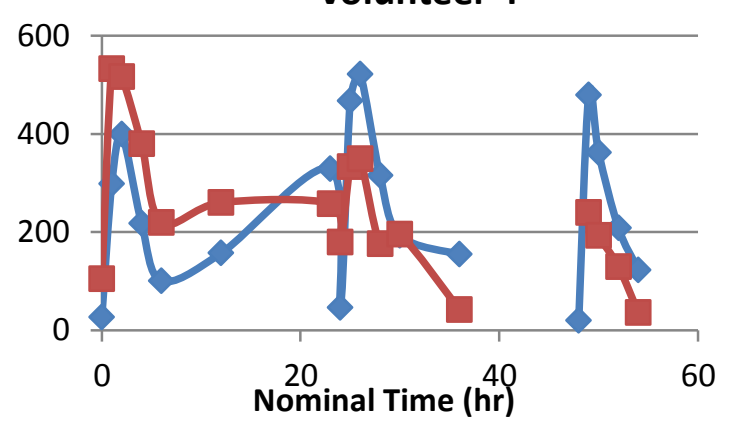

511

Figure 5- A selection of PK profiles from RIA assay concentrations (red squares) and LC-MS/MS method concentrations (blue diamonds). $Y$ axis units are $\mathrm{pg} / \mathrm{mL}$. See supplemental information Figure 6 for the complete set of 9 profiles

\section{Conclusion}

The developed procedure is the first peer reviewed LC-MS/MS method capable of quantifying endogenous levels of glucagon in human plasma. Glucagon levels from healthy volunteers agreed well with the range expected from RIA assays. Our method avoids the radioactivity (and precautions this requires) associated with RIA assays, has a shorter extraction time and good precision and accuracy.

The $25 \mathrm{pg} / \mathrm{mL}$ LLOQ in our qualified assay is a considerable improvement over the lowest LC-MS/MS LLOQ previously reported $(250 \mathrm{pg} / \mathrm{mL})$ in the peer reviewed literature [11]. A 10 $\mathrm{pg} / \mathrm{mL}$ LLOQ has been reported in a conference presentation [14], using a highly sensitive QTRAP mass spectrometer. We were on occasion able to see such levels using our instrument, although we performed the qualification using a $25 \mathrm{pg} / \mathrm{mL}$ LLOQ to improve assay robustness. Transferring this assay on to a more modern instrument may enable the LLOQ of $10 \mathrm{pg} / \mathrm{mL}$ to be achieved routinely. Our 2D extraction procedure was key to achieving such sensitivity, by reducing matrix suppression, background noise, and interferences. To our knowledge this is the first time protein precipitation and size exclusion SPE have been combined for such a purpose for high throughput peptide analysis. Our 
532 surrogate matrix approach, using a mixture of non-extracted surrogate matrix STDs and QCs 533 and extracted authentic matrix QCs, is also a novel strategy for endogenous peptide 534 analysis.

535 Bland-Altman analysis shows a mean positive bias of the LC/MS-MS method versus the RIA 536 that appears to be a concentration-dependent, as would be expected if the RIA was suffering 537 from the hook effect at higher concentrations. The PK profiles from both assays were similar 538 shapes, and both profiles fitted with the nature of the physiological study suggesting the 539 methods are complementary.

540 The assay's performance has been qualified using experiments described in the latest EMA 541 [18] and FDA [17] guidance and in accordance to the principles of GCP [23]. 


\section{Executive Summary}

545

546

547

548

549

550

551

552

553

554

555

556

557

558

559

560

561

562

563

564

565

566

567

568

569

570

571

572

573

574

\section{Introduction}

- Published LC-MS/MS methods are not sensitive enough to quantify endogenous levels of glucagon.

- Endogenous compounds, such as glucagon, can be quantified using either a standard addition, surrogate analyte, or a surrogate matrix approach.

- We favoured the surrogate matrix approach as it avoids extrapolation and is described in the EMA Guideline on bioanalytical method validation.

\section{Results and Discussion}

\section{Method development}

- Extensive optimisation has generated the most sensitive LC-MS/MS method for glucagon quantitation in the peer reviewed literature.

- A novel 2D extraction technique, combining protein precipitation with size exclusion hydrophobic (SEH) SPE, was key to achieving such sensitivity, by reducing matrix suppression, background noise, and interferences.

- Quantitation used a mixture of non-extracted surrogate matrix STDs and QCs and extracted authentic matrix QCs. Such approach is a novel strategy for endogenous peptide analysis.

$\underline{\text { Validation }}$

- Validation experiments performed were based on those described in the latest EMA and FDA guidelines.

- Most experiments, including the precision and accuracy of the method, were within the prospectively defined acceptance criteria.

- However, a degree of plasma sample instability was apparent, and it fell outside of our prospectively defined acceptance criteria.

- The assay is therefore described as qualified, over the range $25-1000 \mathrm{pg} / \mathrm{mL}$, rather than validated. The assay will however be fit-for-purpose for many applications. 


\section{1}

592

- Glucagon levels in healthy volunteers measured by LC-MS/MS showed good agreement with literature values determined by RIA.

- Assessment of assay performance at the 10 and $15 \mathrm{pg} / \mathrm{mL}$ levels allowed the assay LLOQ to be lowered from $25 \mathrm{pg} / \mathrm{mL}$ on a batch to batch basis.

- Reproducible quantitation at the endogenous glucagon level was demonstrated.

\section{$\underline{\text { LC-MS/MS vs. RIA assays for physiological study samples }}$}

- Bland-Altman analysis shows a concentration-dependent positive bias of the LC/MSMS assay versus an RIA, with a mean bias of $+45.06 \mathrm{pg} / \mathrm{mL}$

- Both assays produced similar PK profiles, both of which were feasible considering the nature of the study, and the methods should be regarded as complementary.

\section{Future Perspectives}

We believe that experimentally demanding or troublesome immunoassays, such as the glucagon RIA assay, will increasingly become replaced with LC-MS/MS based methodologies to circumvent issues with cross reactivity, increase sample throughout and avoid the use of radioactivity. To achieve the low LLOQs often required we also believe that approaches such as 2D extraction will become more commonly used. For regulated bioanalytical studies of endogenous compounds, strategies such as surrogate matrix quantitation, which avoids the need to extrapolate the calibration curve, will become the favoured approach. 
The authors have no relevant affiliations or financial involvement with any organization or entity with a financial interest in or financial conflict with the subject matter or materials discussed in the manuscript. This includes employment, consultancies, honoraria, stock ownership or options, expert testimony, grants or patents received or pending, or royalties.

No writing assistance was utilized in the production of this manuscript.

\section{Ethical conduct of research}

The authors state that they have obtained appropriate institutional review board approval (West London Research Ethics Committee: 11/LO/1782) and have followed the principles outlined in the Declaration of Helsinki for all human experimental investigations.

\section{$\underline{\text { References }}$}

1. Kolb A, Rieder S, Born D, et al. Glucagon/insulin ratio as a potential biomarker for pancreatic cancer in patients with new-onset diabetes mellitus. Cancer Biol. Ther.

2. Hinke SA, Pospisilik JA, Demuth HU, et al. Dipeptidyl peptidase IV (DPIV/CD26) degradation of glucagon. Characterization of glucagon degradation products and DPIV-resistant analogs. J. Biol. Chem. 275(6), 3827-34 (2000).

3. Zhu L, Tamvakopoulos C, Xie D, et al. The role of dipeptidyl peptidase IV in the cleavage of glucagon family peptides: in vivo metabolism of pituitary adenylate cyclase activating polypeptide-(1-38). J. Biol. Chem. 278(25), 22418-23 (2003).

4. Tan TM, Field BCT, McCullough $\mathrm{K}$ a, et al. Coadministration of glucagon-like peptide1 during glucagon infusion in humans results in increased energy expenditure and amelioration of hyperglycemia. Diabetes. 62(4), 1131-8 (2013).

5. Taieb J, Mathian B, Millot F, et al. Testosterone measured by 10 immunoassays and by isotope-dilution gas chromatography-mass spectrometry in sera from 116 men, women, and children. Clin. Chem. 49(8), 1381-1395 (2003).

6. FP Alford, SR Bloom J, Nabarro. Glucagon levels in normal and diabetic subjects: Use of a specific immunoabsorbent for glucagon radioimmunoassay. Diabetologia. 13(1), 1-6 (1977).

7. MJ B, Albrechtsen N, Pedersen J, et al. Specificity and sensitivity of commercially available assays for glucagon and oxyntomodulin measurement in humans. Eur $J$ Endocrino. 170(4), 529-38 (2014).

8. Sloan JH, Siegel RW, Ivanova-Cox YT, Watson DE, Deeg M a, Konrad RJ. A novel high-sensitivity electrochemiluminescence $(E C L)$ sandwich immunoassay for the specific quantitative measurement of plasma glucagon. Clin. Biochem. 45(18), 16404 (2012). 
9. Hoofnagle AN, Wener MH. The Fundamental Flaws of Immunoassays and Potential Solutions Using Tandem Mass Spectrometry. J Immunol Methods. 347((1-2)), 3-11 (2009).

10. Delinsky DC, Hill KT, White CA, Bartlett MG. Quantitation of the large polypeptide glucagon by protein precipitation and LC/MS. Biomed. Chromatogr. 18(9), 700-5 (2004).

11. Li YX, Hackman M WC. Quantitation of polypeptides (glucagon and salmon calcitonin) in plasma samples by "high resolution" on a triple quadrupole mass spectrometer. Bioanalysis. 4(6), 685-691 (2012).

12. Veniamin N Lapko, Patrick S Miller, G Paul Brown, Rafiqul Islam, Sarah K Peters, Richard L Sukovaty PFR\& CJK. Sensitive glucagon quantification by immunochemical and LC - MS / MS methods. Bioanalysis. 5(23), 2957-2972 (2013).

13. V. Lapko, P. Brown, R. Nachi, C. Kafonek, A. Dzerk, B. Retke CO, Davis CS and I. Exploring quantification of peptides: measurement of glucagon in human plasma by LC-MS/MS. Presented at: In: EBF 3rd Annual Open Symposium: From Challenges to Solutions. Barcelona, Spain, 1 - 3 December 2010.

14. F. Garofolo, J. N. Mess, L. P. Morin, M. Aiello, X. Misonne, G. Impey, J. Cardenas JM. Glucagon bioanalysis by LC-MS: unprecedented level of sensitivity $(10 \mathrm{pg} / \mathrm{ml})$ for a novel formulation. Presented at: In: 2013 American Association of Pharmaceutical Scientists National Biotechnology Conference. San Diego, CA, 20-22 May 2013.

15. Jones BR, Schultz G a, Eckstein J a, Ackermann BL. Surrogate matrix and surrogate analyte approaches for definitive quantitation of endogenous biomolecules.

Bioanalysis. 4(19), 2343-56 (2012).

16. Bansal SS, Abbate V, Bomford A, et al. Quantitation of hepcidin in serum using ultrahigh-pressure liquid chromatography and a linear ion trap mass spectrometer. Rapid Commun. Mass Spectrom. 24(9), 1251-9 (2010).

17. Guidance for industry: Bioanalytical method validation. U.S. Department of Health and Human Services, Food and Drug Administration, Center for Drug Evaluation and Research (CDER), Center for Veterinary Medicine (CVM), May 2001.

18. Guideline on bioanalytical method validation, EMA. (2012).

19. Lee JW. Method validation and application of protein biomarkers: basic similarities and differences from biotherapeutics. Bioanalysis. 1(8), 1461-74 (2009).

20. Lee JW. Method validation and application of protein biomarkers: basic similarities and differences from biotherapeutics. Bioanalysis. 1(8), 1461-74 (2009).

21. Polaskova V, Kapur A, Khan A, Molloy MP, Baker MS. High-abundance protein depletion: comparison of methods for human plasma biomarker discovery. Electrophoresis. 31(3), 471-82 (2010).

22. Howard JW, Kay RG, Pleasance S, Creaser CS. UHPLC for the separation of proteins and peptides. Bioanalysis. 4(24), 2971-88 (2012). 
23. International committee on harmonisation $(\mathrm{ICH})$ guideline $\mathrm{E} 6$ : Triparite guidelines for GCP, EMEA. (1996).

24. Kreymann B, Williams G, Ghatei MA BS. Glucagon-like peptide-1 7-36: a physiological incretin in man. Lancet. 2(8571), 1300-1304 (1987).

25. Ghatei MA, Uttenthal LO, Bryant MG, Christofides ND, Moody AJ BS. Molecular Forms of Glucagon-Like Immunoreactivity in Porcine Intestine and Pancreas. Endocrinology. (112), 917-923. (1983).

26. Fekete S, Ganzler K, Fekete J. Facts and myths about columns packed with sub-3 microm and sub-2 microm particles. J. Pharm. Biomed. Anal. 51(1), 56-64 (2010).

27. Ruta J, Guillarme D, Rudaz S, Veuthey J-L. Comparison of columns packed with porous sub-2 microm particles and superficially porous sub-3 microm particles for peptide analysis at ambient and high temperature. J. Sep. Sci. 33(16), 2465-2477 (2010).

28. Ismaiel OA, Zhang T, Jenkins R, Karnes HT. Determination of octreotide and assessment of matrix effects in human plasma using ultra high performance liquid chromatography-tandem mass spectrometry. J. Chromatogr. B. Analyt. Technol. Biomed. Life Sci. 879(22), 2081-2088 (2011).

29. Holčapek M, Jirásko R, Lísa M. Recent developments in liquid chromatography-mass spectrometry and related techniques. J. Chromatogr. A. 1259, 3-15 (2012).

30. Kay R, Barton C, Ratcliffe L, et al. Enrichment of low molecular weight serum proteins using acetonitrile precipitation for mass spectrometry based proteomic analysis. Rapid Commun. Mass Spectrom. 22(20), 3255-60 (2008).

31. Halquist MS, Karnes HT. Quantification of Alefacept, an immunosuppressive fusion protein in human plasma using a protein analogue internal standard, trypsin cleaved signature peptides and liquid chromatography tandem mass spectrometry. J. Chromatogr. B. Analyt. Technol. Biomed. Life Sci. 879(11-12), 789-98 (2011).

32. Wang Y, Qu Y, Bellows CL, Ahn J, Burkey JL, Taylor SW. Simultaneous quantification of davalintide, a novel amylin-mimetic peptide, and its active metabolite in beagle and rat plasma by online SPE and LC-MS/MS. Bioanalysis. 4, 2141-2152 (2012).

33. Chappell D, Lee A, Castro-Perez J, et al. An ultrasensitive method for the quantitation of active and inactive GLP-1 in human plasma via immunoaffinity LC-MS/MS.

Bioanalysis. 6(1), 33-42 (2014).

34. Omenn GS. THE HUPO Human Plasma Proteome Project. PROTEOMICS - Clin. Appl. 1(8), 769-779 (2007).

35. Houghton R, Horro Pita C, Ward I, Macarthur R. Generic approach to validation of small-molecule LC-MS/MS biomarker assays. Bioanalysis. 1(8), 1365-74 (2009).

36. Rai AJ, Gelfand CA, Haywood BC, et al. HUPO Plasma Proteome Project specimen collection and handling: towards the standardization of parameters for plasma proteome samples. Proteomics. 5(13), 3262-77 (2005). 
37. Green BD, Flatt PR, Bailey CJ. Dipeptidyl peptidase IV (DPP IV) inhibitors: A newly emerging drug class for the treatment of type 2 diabetes. Diab. Vasc. Dis. Res. 3(3), 159-65 (2006).

38. Leboeuf R, Langlois M-F, Martin M, Ahnadi CE, Fink GD. "Hook effect" in calcitonin immunoradiometric assay in patients with metastatic medullary thyroid carcinoma: case report and review of the literature. J. Clin. Endocrinol. Metab. 91(2), 361-4 (2006). 